\title{
Luces y sombras del capital en el siglo xxI: las desigualdades del capitalismo patrimonial
}

\section{Lights and shadows of capital in the XxI century: the inequalities of patrimonial capitalism}

PiketTy, Thomas (20 I4), EL CAPITAL EN EL SIGLO XXI, Fondo de Cultura Económica, MéXico, 663 PP., ISBN. 978-607-i 6-24I 6-I.

El libro del economista francés Thomas Piketty irrumpió en los círculos académicos y políticos de manera inusitada. Su publicación ha despertado un gran interés editorial y del público en general, fenómeno que regularmente no ocurre tratándose de una obra cuya temática es la economía. El autor estudió en Francia y es profesor en la Escuela de Economía de París, asimismo ha sido acreedor a distintos reconocimientos internacionales. Ser un experto en la materia le ha permitido, de manera eficiente, abordar temas como el comportamiento del capitalismo contemporáneo y las posibles soluciones frente a las desigualdades sociales inherentes a él. Esto se relaciona con el problema de la distribución del ingreso planteado por David Ricardo (1958).

El panorama en el que se inscribe esta investigación es la crisis mundial de 2008, desatada en Estados Unidos y no resuelta aún, especialmente en la Unión Europea; estos países enfrentan una creciente disparidad de los ingresos entre los percentiles superiores e inferiores (que son la mayoría de la población).

Este libro es un best seller, lo que pareciera extraño tratándose de un texto de economía; no obstante, la actualidad del tema que se expone y el tratamiento que le da el autor ha despertado el interés de muchos lectores, pues aborda el escenario actual caracterizado por la extrema desigualdad social no sólo en la periferia sino en el centro del sistema económico político mundial: "99\%, frente a 1\%" de la población internacional concentra escandalosamente la riqueza generada. Obviamente, esto se encuentra en consonancia directa con las movilizaciones sociales (indignados, ocupas, etcétera), que han surgido como respuesta a quienes han desencadenado esta crisis económica (corporaciones trasnacionales y gobiernos de los países ricos).

La obra está integrada por cuatro partes (16 capítulos). En la primera plantea un marco donde analiza los conceptos de ingreso nacional, capital 
y capital/ingreso, ejes teóricos de esta investigación; en la segunda parte se expone la dinámica de la relación capital/ingreso, así como la distribución del ingreso entre el trabajo y el capital. En la tercera trata la estructura de la desigualdad como su objetivo principal; en la cuarta parte plantea su propuesta práctica en torno a la "regulación del capitalismo en el siglo XxI", que gira alrededor del impuesto al capital y a las grandes fortunas, como parte de las acciones prácticas y normativas hacia un nuevo Estado social.

\section{El contexto y metodología en la propuesta de Piketty}

De manera acertada, el autor destaca que los problemas fundamentales del capitalismo contemporáneo (p. 30) tienen en las crecientes desigualdades sociales, la dificultad básica del análisis económico en el inicio del siglo XXI (al igual que las preocupaciones políticas de los gobiernos), lo que resulta fundamental, puesto que "Al principio de la década de 2010, la participación de $10 \%$ de los patrimonios más elevados se sitúa en torno a $60 \%$ de la riqueza nacional en la mayoría de los países europeos” (p. 283). La gran importancia del tema que trata este autor puede reafirmarse con las declaraciones de la presidente de la Reserva Federal de Estados Unidos (FED), Janet Yellen, en el sentido de que esta institución apoya diversas investigaciones para conocer cuáles son las políticas que reducirán las desigualdades sociales (Forbes, 2015). Lo que no deja de ser sorprendente, es dicho desconocimiento por parte del gobierno de la economía más implicada en este problema.

La metodología empleada se basa en abundantes datos estadísticos que se despliegan en un novedoso anexo que puede ser consultado en la página piketty@ens.fr, y "que permiten estudiar la evolución a muy largo plazo del valor total del acervo de la riqueza nacional (ya sea que se trate del capital en forma de tierras, inmobiliario, industrial o financiero) medido en número de años de ingreso nacional del país considerado. El estudio de esa relación capital/producto o capital ingreso de manera global, es un ejercicio que tiene límites". Asimismo se argumenta que las fuentes sucesorias y patrimoniales posibilitan estudiar "la evolución de la importancia respectiva de la herencia y del ahorro en la constitución de los patrimonios y en la dinámica de la desigualdad de la riqueza" (p. 34).

Se toman y se comparan los casos de Estados Unidos y Francia, principalmente, así como de los países ricos en general. Evidentemente, se requiere de conceptos adecuados, por lo que las estadísticas no explican ni demuestran nada por sí mismas, sin embargo permiten combinar historia con economía y sus conceptos. 
Puede adelantarse que, a pesar de que Piketty está convencido de que su explicación teórica es acertada -aunque no es suficientemente clara-, es útil de cualquier forma para entender la dinámica de la economía actual, sobre todo en cuanto a las conclusiones políticas a las que llega y que han despertado gran interés en los lectores; también lo es por revalorar el análisis estadístico, siguiendo a Kuznets, para retroalimentarse con sus investigaciones.

En esta obra de 663 páginas, más el anexo estadístico, destacan principalmente dos grandes preocupaciones que corresponden a su explicación teórica y a su propuesta concreta frente al problema estudiado:

1. La situación de desigualdad económica y social patente en el mundo se explica básicamente a partir de lo que el autor llama la "ley fundamental del capitalismo", consistente en que la tasa de rendimiento del capital aumenta más rápido que el crecimiento económico (medido por el PIB); es decir, estas dos variables estan íntimamente interrelacionadas según el autor; lo cual es cierto, pero también se debe reconocer que, en el fondo, ambas son consecuencia de las formas histórico-concretas de acumulación de capital (ésta como variable independiente), por lo que no se trata de una simple relación de causa-efecto entre ellas.

Aquí se apunta hacia algo fundamental que niega la economía neoclásica, ya que aún en la búsqueda del equilibrio general los mercados se tropiezan con la inefable contraposición capital/ingreso, apuntando a la relación capital/trabajo como la fuente primaria de las desigualdades mencionadas.

En consecuencia, están presentes evidentes similitudes con Keynes y Marx a partir de la presencia y persistencia del desequilibrio básico del capitalismo, que se explica de manera diferente: oferta global/demanda efectiva, según Keynes; o mediante las contradicciones internas de la ley de la tendencia descendente de la tasa de ganancia, de acuerdo con Marx. Así pues, destaca sobremanera la presencia de un diálogo subrepticio con El capital de Marx, en especial frente a esta última tendencia, que él interpreta mejor como el incremento de la tasa de rendimiento del capital; empero, la primera se expresa a la vez como un incremento de la masa de ganancias, tanto como del capital total.

2. La propuesta cardinal para resolver el desequilibrio citado es a través de un impuesto progresivo al capital (incluyendo el patrimonio). Sin embargo, si se profundiza la desigualdad primaria, pero sobre todo al privilegiarse la acumulación ilimitada de capital, esta medida puede no tener el efecto deseado.

Pero además, tales impuestos son un arma de doble filo: permiten aumentar la recaudación, pero simultáneamente atentan contra la tasa de 
inversión (y por lo tanto del ahorro), que constituye el factor más dinámico de la acumulación de capital y el crecimiento económico.

\title{
La dinámica del capital y el ingreso
}

Pikkety comienza con una pregunta crucial para abordar su planteamiento:

\begin{abstract}
¿Acaso la dinámica de la acumulación del capital privado conduce inevitablemente a una concentración cada vez mayor de la riqueza y del poder en unas cuantas manos, como lo creyó Marx en el siglo xIx? O bien, ¿acaso las fuerzas equilibradoras del crecimiento, la competencia y el progreso técnico conducen espontáneamente a una reducción y a una armoniosa estabilización de las desigualdades en las fases avanzadas del desarrollo, como lo pensó Kuznets en el siglo Xx?
\end{abstract}

Y luego advierte que: "El crecimiento moderno y la difusión de los conocimientos permitieron evitar el apocalipsis marxista, más no modificaron la estructuras profundas del capital y de las desigualdades" (p. 15).

Así pues, puede decirse de entrada, que su posición teórico-metodológica se sitúa entre Marx y Kuznets. El primero, advirtió la tendencia del capitalismo a agravar sus contradicciones internas (que se reflejan en la desvalorización del capital a consecuencia del incremento de la productividad del trabajo), mientras que el segundo llegó a proponer que las desigualdades aminoraban con la marcha del crecimiento económico y las leyes del mercado.

Empero, Piketty apunta que Marx considera el "principio de la acumulación infinita [...] Sin límite natural [...] Marx pasó totalmente por alto de un progreso técnico y de un crecimiento continuo de la productividad" (p. 23) y "no se cuestionó cómo sería la organización política y económica de una sociedad en la que se hubiera abolido por completo la propiedad privada." (p. 23). Pero esto elude al hecho de que la hipótesis básica en la obra de Marx (1985) es precisamente el incremento de las fuerzas productivas y, por lo tanto, de la productividad del trabajo. Por otro lado, "Según las teorías de Kuznets, en efecto, la desigualdad de los ingresos se ve destinada a disminuir en las fases avanzadas del desarrollo capitalista, sin importar las políticas seguidas o las características del país, y luego tiende a estabilizarse en un nivel aceptable" (p. 25). Y señala que todo converge con el "sendero del crecimiento equilibrado" de Robert Sollow (del que pueden mencionarse sus similitudes con el concepto actual de desarrollo sustentable).

Además, destaca que Kuznets advirtió que entre 1913 y 1948, en Estados Unidos, se dio una fuerte reducción de las desigualdades en los ingresos y agrega que desde 1970 la desigualdad creció significativamente 
en los países ricos; donde de 2000-2010 la concentración de ingresos superó el nivel récord de 1910-1920 (p. 25).

Pero su aporte teórico más bien está en otro lado, y es la botella que lanza al mar del debate, con su mensaje principal consistente en que las riquezas acumuladas en el pasado adquieren una importancia considerable "potencialmente desmedida y desestabilizadora" (p. 24).

El hilo conductor del análisis son los procesos que reducen o aumentan las desigualdades. En el primer caso destacan las fuerzas que conducen a la convergencia: difusión de los conocimientos y de inversión en la capacitación y formación de habilidades, es decir, capital humano y el reemplazo de la lucha de clases por la "guerra de edades" (p. 37). En el segundo caso, se refiere a las fuerzas opuestas o de divergencia: un mundo con lento crecimiento y elevado rendimiento del capital, que constituyen la principal amenaza para la distribución de la riqueza a largo plazo (p. 38).

De esa manera, afirma que el hecho de que el rendimiento del capital sea mayor que el crecimiento económico es el principal obstáculo a la redistribución de la riqueza, señalando correctamente que esto no representa una imperfección del mercado (p. 43), lo que constituye una postura crítica frente al neoliberalismo.

Sin embargo, parecería que esa ley queda reducida en su impacto negativo en la esfera internacional al señalar de manera muy optimista que "al adoptar los medios de producción y al alcanzar los niveles de cualificación de los países ricos los países menos desarrollados reducen su retraso en productividad y hacen progresar sus ingresos" (p. 37). Esto, como consecuencia de compartir el saber, más que de los mecanismos de mercado. En este punto, puede mencionarse el rol que desempeña la sociedad del conocimiento.

Lo verdaderamente importante radica en la siguiente afirmación: "En las sociedades de bajo crecimiento, la riqueza originada en el pasado adquiere naturalmente una importancia desproporcionada, pues basta con un bajo flujo de ahorro nuevo para incrementar de manera continua y sustancial el acervo de riqueza" (p. 38), lo cual se debe a que "la acumulación y la distribución de la riqueza estarán dominadas hoy día ya no por un implacable enfrentamiento entre herederos y los desheredados sino más bien por una lógica de ahorro de ciclo de vida: cada individuo acumula un patrimonio para su vejez. Entonces, el adelanto médico y la mejoría de las condiciones de vida transformarían totalmente la naturaleza del capital" (p. 37). 


\section{El origen de las desigualdades en el capitalismo patrimonial}

La parte medular del libro se halla en la relación capital/ingresos que se obtiene dividiendo el capital entre el flujo anual de ingresos (p. 65), lo cual permite asociar el acervo del capital con el flujo de ingresos del mismo capital. La relación capital/ingresos $(\beta)$, se vincula de manera simple con la participación de los ingresos del capital en el ingreso nacional". ${ }^{1}$

Tres conceptos básicos están presentes en dicha explicación: la relación capital/ingreso, la relación del capital en el ingreso y la tasa de rendimiento del capital, que en su conjunto determinan la distribución del ingreso.

La tesis fundamental del libro consiste en que "una diferencia aparentemente limitada entre la tasa de rendimiento del capital y la tasa de crecimiento puede producir a largo plazo efectos muy potentes y desestabilizadores en la estructura y la dinámica de las desigualdades en una sociedad determinada" (pp. 92-93).

Con esta premisa acaba con los maltusianos: "Un fuerte incremento demográfico tiende a desempeñar un papel igualador, puesto que disminuye la importancia de la riqueza originada en el pasado y por consiguiente de las herencias; de cierta manera, cada generación debe construirse a sí misma” (p. 110). Y también con la growthmania: "Un bajo crecimiento anual acumulado durante un periodo muy largo lleva un incremento considerable" (p. 91). Esto es muy simple de comprender, pero más importante es su insistencia en que basta con sólo el uno por ciento de crecimiento, para que una sociedad se renueve profundamente (p. 112), lo cual es un planteamiento fundamental que es necesario rescatar, porque demuestra que el crecimiento no es un mero asunto cuantitativo sino también cualitativo.

Destaca igualmente: "Sin duda el crecimiento económico es por sí mismo incapaz de satisfacer esa esperanza democrática y meritocrática, que debe fundarse en instituciones específicas para ese propósito y no sólo en las fuerzas del progreso técnico y del mercado" (p. 113).

Sin embargo, afirma que el crecimiento permitió una considerable mejora en las últimas décadas (p. 110). Esto es cierto, pero a costa de la degradación social y ambiental, lo que no menciona; reconoce que el crecimiento puede llevar a nuevas formas de desigualdades y, al mismo tiempo, que la desigualdad de los patrimonios sea más importante y la herencia menos determinante (p. 184).

\footnotetext{
${ }^{1}$ Se utiliza la fórmula $\alpha=r \times \beta$; se señala que se trata de "una simple igualdad contable" (p. 67), donde la participación del ingreso en el capital se expresa en $\alpha, r$ expresa la tasa de rendimiento del capital y $\beta$ la relación capital ingreso.
} 
A continuación plantea lo que denomina la segunda ley fundamental del capital, ${ }^{2}$ que vincula la tasa de ahorro con el crecimiento y con la relación capital/ingreso señalando que en una sociedad, prácticamente estancada desde el punto de vista económico, las riquezas acumuladas en el pasado adquieren inevitablemente una importancia desmedida (y por lo tanto más ahorro). El resultado es la emergencia de un nuevo "capitalismo patrimonial” (p. 161); ${ }^{3}$ y que desde luego se expresa en el papel del consumo; sin embargo, ello es relegado en su estudio. Empero, reconoce dentro de la relación capital/ingreso la importancia cardinal de la relación capital/trabajo (p. 255), acotando que en el mundo actual la lucha de clases ha sido sustituida por la lucha entre percentiles (p. 276).

Lo siguiente resulta una explicación adecuada para entender el comportamiento económico del siglo xx: "El desarrollo de una verdadera clase media patrimonial constituye la principal transformación estructural de la distribución de la riqueza en el siglo xx" (p. 285). No obstante, la jerarquía de los ingresos se encuentra dominada por los muy altos ingresos del trabajo y no por los heredados", afirmando que la sociedad de los rentistas es desplazada por la sociedad de los ejecutivos (p. 289).

Posteriormente, en torno a la crisis financiera de 2008 y su relación con la desigualdad social, afirma que ésta tuvo como consecuencia un casi estancamiento del poder adquisitivo de las clases populares y medias en Estados Unidos de norteamérica, lo que sólo incrementó la tendencia a un creciente endeudamiento de los hogares modelos.

En este contexto, reconoce atinadamente que la democratización del sistema escolar no redujo la desigualdad de calificación, y por consiguiente no permitió aminorar las de los salarios (p. 335). Sin embargo, destaca que la mejor manera de combatir la desigualdad es con el impulso a la educación (p. 336). Y señala que la educación y la tecnología son fuerzas fundamentales para la formación y aumento de los salarios, así como para la reducción de las desigualdades salariales (p. 367), por estas razones, emprende la crítica a la teoría de la productividad marginal (p. 363).

Como resultado de su investigación encuentra que: "En concreto, eso significa que los patrimonios resultantes del pasado se recapitalizan mucho más rápido que el crecimiento de la economía, incluso en ausencia de cualquier ingreso del trabajo" (p. 388). Dentro de su perspectiva teórica esto se explica por el hecho, reiterado en su obra, de que la tasa de rendi-

\footnotetext{
${ }^{2} \beta=s / g$ Describe la vinculación de la tasa de ahorro con el crecimiento con la relación capital/ ingreso $(\beta)$ y el crecimiento $(g)$.

${ }^{3}$ Incluye bajo el concepto de patrimonio: $a$ ) bienes inmuebles, $b$ ) activos financieros (depósitos bancarios, planes de ahorro, cartera de acciones y obligaciones, contratos de seguro de vida, fondos de pensiones, netos de deuda) (p. 283).
} 
miento del capital (que incluye todo tipo de rentas) tiende a crecer más rápido que el crecimiento económico.

\section{Solución a las desigualdades: impuesto progresivo al capital}

Piketty inicia este punto crucial de su planteamiento mencionando que, hasta la primera guerra mundial, en la mayoría de los países no existía un impuesto a los ingresos del capital (p. 574). Y explica que la principal innovación del siglo xx en la materia fiscal fue la creación y desarrollo del impuesto progresivo sobre el ingreso.

Para abordar esta cuestión, parte de una conclusión fundamental la cual se desprende de su análisis, es que: "sería ilusorio imaginar que, en la estructura del crecimiento moderno, o en las leyes de la economía de mercado, existen fuerzas de convergencia que conduzcan de forma natural a una reducción de la desigualdad patrimonial o a una armoniosa estabilidad" (p. 414).

La clave de ello se resume en que un mayor rendimiento del capital en relación al crecimiento significa que "el pasado tiende a devorar el presente", ya que "en todas las sociedades existen dos formas de poder vivir con holgura: por medio del trabajo o por medio de la herencia" (p. 476), aunque en realidad, ambas posibilidades dependen más bien del acceso a la propiedad o no de los medios de producción; sin embargo, anticipa que la herencia seguramente recobrará una importancia cercana a la que tuvo en el siglo XIx.

Luego se pregunta si la renta es enemiga de la racionalidad económica (p. 465) y está de acuerdo con ello; no obstante, el capitalista vive del derroche más que de la abstinencia.

Ofrece asimismo una abrumadora cantidad de datos estadísticos que reflejan la realidad de la desigualdad: "Hoy en día, la participación del milésimo superior es aparentemente de casi $20 \%$ de la riqueza total y la del percentil superior sólo puede situarse entre 80 y $90 \%$; la mitad inferior de la población mundial posee sin duda alguna el 5\% del patrimonio total" (p. 482).

Finalmente, propone que "la institución ideal para evitar una espiral desigualitaria sin fin y retomar el control de la dinámica en curso sería un impuesto mundial y progresivo sobre el capital", ya que es una herramienta más apropiada para responder a los retos del siglo xxi.

Concluye en que la redistribución moderna, no consiste en transferir las riquezas de los ricos a los pobres, o por lo menos no de manera explícita; reside, en cambio, en financiar servicios públicos e ingresos de reposición más o menos iguales para todos, pero sobre todo en el ámbito de la educación, la salud, y las jubilaciones (p. 528). 
Más que la aplicación del impuesto progresivo, el más serio aporte del libro es la propuesta de creación de nuevas formas de propiedad y control democrático del capital (p. 639), lo que es correcto dada la prevalencia del economicismo; asimismo "se debe partir del principio de que las formas concretas de la democracia y del capital siempre tiene que estarse reinventando" (p. 641).

\section{Conclusión: el futuro del capitalismo y las desigualdades}

En suma, Piketty afirma que el crecimiento económico (que incluye cambios en el ingreso y el capital) depende de un crecimiento mayor del capital y su rendimiento para que así tal crecimiento se sostenga, aunque fuese al uno por ciento (p. 112). Esta aserción es importante, ya que el crecimiento sostenido es la panacea para el mainstream del pensamiento económico contemporáneo

No obstante, con sus mismos datos se demuestra en realidad que el sobreconsumo de la Unión Europea (con énfasis en la zona Euro) antecedió a la crisis mencionada. Lo mismo aconteció en EUA, donde la reducción de las desigualdades en 1950-1970 permitió la fase más igualitaria de su historia, mientras que de 1970-1980 se asiste a "una explosión sin precedente en la desigualdad en los ingresos de EUA" (p. 321).

Puede resultar una obviedad la forma como él explica la perturbación principal del capitalismo, pero Piketty lo hace desde el ángulo de la distribución, la cual sin embargo no hace sino expresar un conflicto mayor como es el existente entre la expansión tecnológica y la valorización del capital; lo que ocurre es que la contradicción producción-consumo, no es más que una derivación de aquel conflicto fundamental.

Por su parte, las dificultades de esta obra tienen que ver con: 1 . Percibir al capital como sinónimo de riqueza, ya que hay que distinguir la riqueza material, que son los valores de uso (en última instancia provienen de la naturaleza), de la riqueza social, que está constituida por valoresmercancías (expresados en dinero), una de cuyas partes representa capital, la otra la renta; 2. La forma en que define al capital, como el conjunto de los activos no humanos que pueden ser poseídos o intercambiados (p. 60), hace a un lado al capital productivo y considera todo flujo de dinero como un activo (ya que puede ser pasivo), igualmente entiende el capital como un acervo, un stock, sin tomar en cuenta que el capital circulante (materias primas, fuerza de trabajo) constituye un flujo (y no deja de serlo en tanto esté transitoriamente en almacenamiento); 3. Minimizar la crisis ambiental y el cambio climático (p. 633).

La limitación principal de Piketty es que no toma en cuenta que la lógica del capital requiere de dichas desigualdades para sostenerse y per- 
durar, por lo que una mejor redistribución del ingreso para conveniencia social y política que no toca las relaciones de producción y de propiedad es pasajera e ilusoria y conduce inevitablemente a una mayor desigualdad. Así entonces, dichos impuestos, sin tocar asuntos como el armamentismo, consumismo, productivismo y la depredación socioambiental, no lograrían aminorar las desigualdades y en cambio sí incrementar a la larga su primera ley fundamental.

Desde luego es importante destacar sus aportes, aunque su propuesta en cierta forma viable resulta poco ingenua, ya que lejos de resolver el desequilibrio estudiado lo puede agudizar a la larga, por lo que su efecto es tan sólo de corto plazo y limitado siempre y cuando no esté presente una verdadera reapropiación social del capital, sino tan sólo una regulación de éste (con el riesgo de quedarse en el papel).

Finalmente, la mejor forma de lograr aminorar la causa del desequilibrio fundamental descrito por Piketty es mediante la aplicación de impuestos al capital y la herencia, así como el control social del mismo, todo lo cual requiere de formas de propiedad novedosas.

Pero lo más importante en este libro radica en que "La función principal del impuesto sobre el capital no es financiar al Estado Social, sino regular el capitalismo" (p. 277), ésta es una propuesta clave para la crítica del neoliberalismo en crisis para encontrar soluciones democráticas.

Por estas razones, este es un libro básico para conocer y debatir las transformaciones ocurridas en el capitalismo hasta las primeras décadas del siglo XXI, y es altamente recomendable para vislumbrar los posibles caminos que tiene una sociedad altamente dinámica en los aspectos políticos y sociales; aunque hasta ahora incapaz de frenar las desigualdades (a nivel local-global), así como los conflictos crecientes derivados de ellas.

\section{Fuentes consultadas}

Forbes (2015), “Janet Yellen quiere más investigación sobre desigualdad en eu", Forbes, México, 2 de abril 2015, <www.forbes.com.mx/ janet-yellen-quiere-mas-investigación-sobre-desigualdad-en-eu/>, 15 de abril de 2015 .

Recibido: 3 de junio de 2015. Aceptado: 3 de agosto de 2015.

Guillermo Torres-Carral. Doctor en ciencias agrícolas por la Universidad Agrícola de Varsovia, Polonia. Egresado de la Facultad de Economía de la Universidad Nacional Autónoma de México. Se desempeña como 
profesor-investigador de tiempo completo en el Departamento de Sociología Rural de la Universidad Autónoma Chapingo, en los programas educativos del doctorado de Ciencias Agrarias, de la maestría (y licenciatura) en sociología rural y del doctorado en educación agrícola superior. Es miembro, desde 1992, del Sistema Nacional de Investigadores, nivel II. Sus líneas de investigación son Economía ecológica, economía, sociedad y desarrollo, nueva ruralidad y desarrollo sustentable en México. Entre sus publicaciones más recientes se encuentran: Poscivilización: guerra y ruralidad, Plaza y Valdés-Universidad Autónoma de Chapingo, México (2006); El desarrollo sustentable en México, Plaza y Valdés-Universidad Autónoma de Chapingo, México (2009); Desarrollo compatible: nueva ruralidad y nueva urbanidad, Plaza y Valdés-Universidad Autónoma de Chapingo, México (2012); El Tao de la economía. La economía política del ambiente, Plaza y Valdés-Universidad Autónoma de Chapingo, México (2015); "Territorialidad y sustentabilidad urbana en la ZMvM", Economía, Sociedad y Territorio, XI (36), El Colegio Mexiquense, A. C., Zinacantepec, pp. 317-347 (2011); "Los límites del antropocentrismo de Marx", Ensayos y artículos de Sociología Rural, núm. 13, Universidad Autónoma de Chapingo, Sociología Rural, México, pp. 20-33 (2014); "La economía política de la guerra", Pacarina del Sur. Revista de Pensamiento Crítico Latinoamericano, núm. 19, en línea (2014); "El retorno de Karl Marx y la crítica de la economía ecológica", Revista Pensamiento al margen, núm. 2, en línea (2015); "Armamentismo y sobreconsumo en el capitalismo contemporáneo. La economía política de la guerra”, Revista Economía: Teoría y Práctica, núm. 38, Universidad Autónoma Metropolitana, México, pp. 149-181 (2013). 